\title{
Microarray Expression Profile of Circular RNAs in Plasma from Primary Biliary Cholangitis Patients
}

\author{
Jiajia Zheng ${ }^{\mathrm{a}}$ Zhenrong Li ${ }^{\mathrm{a}} \quad$ Tiancheng Wang $^{\mathrm{a}} \quad$ Yang Zhao $^{\mathrm{a}} \quad$ Yongfeng Wang ${ }^{\mathrm{b}}$ \\ aDepartment of Laboratory Medicine, Peking University Third Hospital, 49 North Garden Road, Haidian \\ District, Beijing, bepartment of Laboratory Medicine, The First Affiliated Hospital of Zhengzhou \\ University, 1 East Jianshe Road, Zhengzhou, Henan, P R China
}

\author{
Key Words \\ CircRNAs • Hsa_circ_402458 PBC
}

\begin{abstract}
Background/Aims: Circular RNAs (circRNAs) play a crucial role in the occurrence of several diseases, including autoimmune diseases. However, their role in primary biliary cholangitis $(\mathrm{PBC})$ remains unclear. Here, we aimed to determine the circRNA expression profile in plasma from PBC patients and further explore the value of circRNA in diagnosing PBC. Methods: CircRNA microarrays were used to determine circRNA expression profiles in plasma samples from 6 PBC patients and 6 healthy controls. Statistical analyses identified differentially expressed circRNAs, and these circRNAs were verified by qRT-PCR in 29 PBC patients and 30 healthy controls. MicroRNA (miRNA) target prediction software identified putative miRNA response elements (MREs), which were used to construct a map of circRNA-miRNA interactions for the differentially expressed circRNAs. Results: Our results indicated that there were 18 up-regulated and 4 down-regulated circular RNAs in the plasma from PBC patients compared with that from healthy individuals. Among the differentially expressed circRNAs, hsa_circ_402458 $(P=0.0033)$, hsa_circ_087631 and hsa_circ_406329 $(P=0.0185)$ were up-regulated, and hsa_circ_407176 $(P=0.0066)$ and hsa_circ_082319 were down-regulated in the PBC group versus the healthy group as demonstrated by qRT-PCR. In particular, hsa_circ_402458 was significantly higher in PBC patients not receiving UDCA treatment than in PBC patients receiving UDCA treatment $(P=0.0338)$. The area under the receiver operating characteristic curve for hsa_circ_402458 for diagnosing PBC was $0.710(P=0.005)$. For hsa_circ_402458, two putative miRNA targets, hsamiR-522-3p and hsa-miR-943, were predicted. Conclusions: circRNA dysregulation may play a role in PBC pathogenesis, and hsa_circ_402458 shows promise as a candidate biomarker for PBC.

(C) 2017 The Author(s)

Published by S. Karger AG, Basel
\end{abstract}

\section{Introduction}

Primary biliary cholangitis (PBC), previously known as primary biliary cirrhosis, is a rare chronic autoimmune cholestatic liver disease characterized by lymphocytic cholangitis

Jiajia Zheng

and Yongfeng Wang

KARGER
Department of Laboratory Medicine, Peking University Third Hospital, Beijing,

Department of Laboratory Medicine, The First Affiliated Hospital of Zhengzhou

University, Zhengzhou, (P R China)

E-Mail gingkoblue@163.com, wangyongfeng@bjmu.edu.cn 


\section{Cellular Physiology Cell Physiol Biochem 2017;44:1271-1281 \begin{tabular}{l|l} 
and Biochemistry Published online: November 29, 2017 & $\begin{array}{l}\text { C } 2017 \text { The Author(s). Published by S. Karger AG, Basel } \\
\text { www.karger.com/cpb }\end{array}$
\end{tabular} \\ Zheng et al.: Hsa_Circ_402458 is Upregulated in Primary Biliary Cholangitis}

and gradual destruction of the small intrahepatic bile ducts, which leads to fibrosis; PBC may also lead to cirrhosis, which is caused by its resulting complications. PBC is mainly diagnosed in women, with a female to male ratio of approximately 10 to $1[1,2]$. The pathogenesis of $\mathrm{PBC}$ is complex and involves genetic, environmental, autoimmune and other factors; its precise mechanism has not yet been fully elucidated. Ursodeoxycholic acid (UDCA) is the established first-line therapy and the current standard of care for PBC. However, up to $40 \%$ of patients have an incomplete biochemical response to UDCA, and this has been shown to be associated with the risk of progression to end-stage liver disease and worse transplantfree survival rates than those in UDCA-responsive patients [3-5]. PBC is usually hard to detect in asymptomatic patients until the disease progresses to a more severe stage; at the severe stage, serum liver function screening and UDCA therapy are commonly used. What is worse, most asymptomatic patients become symptomatic and may die or require a liver transplant within 5 years of not receiving an intervention [6, 7]. Overall survival rates in these asymptomatic patients are shorter than those predicted for age- and gender-matched control populations. The median survival is only $6 \sim 10$ years [8]. At present, histological staging is used to confirm PBC diagnoses and stratification; however, an ideal non-invasive molecular marker for diagnosing PBC in its early stages, assessing its disease progression and determining treatment efficacy is lacking.

Circular RNAs (circRNAs) are a novel type of universal and diverse endogenous noncoding RNA (ncRNA); unlike linear RNAs, they form covalently closed continuous loops and act as gene regulators in mammals [9]. CircRNAs are differentially generated by backsplicing exons or from lariat introns. The 3' and 5' ends normally present in an RNA molecule can be joined together by covalent bonds; this leads to circularization, resistance to RNA exonucleasemediated degradation, and high stability $[10,11]$. Interestingly, circRNAs are abundant, evolutionally conserved and relatively stable in the cytoplasm; these properties indicate that circRNAs may be more suitable biomarkers than other types of RNA [12]. CircRNAs play important roles as miRNA sponges or interact with RNA-associated proteins to form RNAprotein complexes that regulate gene transcription [13, 14]. CircRNAs have been identified in exosomes, and more recently, chromosomal translocation in cancer has been shown to generate aberrant fusion-circRNAs associated with resistance to drug treatments $[15,16]$. In addition, though they were originally thought to be non-coding, there is now increasing evidence to suggest that select circRNAs can be translated into functional proteins [17]. Although much remains to be elucidated regarding circRNA biology and their mechanisms of gene regulation, emerging evidence has revealed the function of circRNAs in various cancers [18] and other human diseases, such as cardiovascular disease, Alzheimer's disease, and diabetes [19-21]; thus, circRNAs may potentially serve as novel biomarkers and therapeutic targets for treating such diseases.

In the present study, we identified a small number of circRNAs that are up- or downregulated in the plasma from PBC patients; these circRNAs may have important functions in PBC development and progression. Importantly, increased expression levels of hsa circ_402458 in plasma from PBC patients may serve as a sensitive and specific biomarker for diagnosing PBC patients.

\section{Materials and Methods}

\section{Patients and Blood samples}

The subjects studied herein were classified into two cohorts; the cohort for circRNA microarray analysis included 6 PBC patients and 6 healthy controls. The cohort for the real-time PCR analysis included 29 PBC patients and 30 healthy individuals. Diagnoses for all patients were based on the established criteria for PBC [3]. All individuals enrolled in this study were were subjected to serum liver function, autoimmune antibody and immunoglobulin assays and liver ultrasounds. HBsAg and anti-HCV were also tested to exclude the possibility of HBV and HCV infection. The clinical features of this cohort are presented in Table 1 . The majority (26/29) of PBC cases were women, and 83\% (24/29) received ursodeoxycholic acid therapy as 


\section{Cellular Physiology Cell Physiol Biochem 2017;44:1271-1281

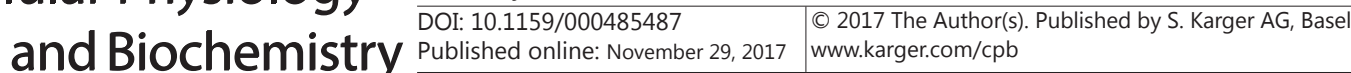 \\ Zheng et al.: Hsa_Circ_402458 is Upregulated in Primary Biliary Cholangitis}

Table 1. Clinical characteristics of PBC patients and Healthy controls. UDCA=Ursodeoxycholic acid;* Biochemical response to UDCA was determined by Paris II criteria [3]

\begin{tabular}{ccc}
\hline & $\mathrm{PBC}(\mathrm{n}=29)$ & Healthy Controls(n=30) \\
\hline Mean age,range & $58(35-85)$ & $45(25-65)$ \\
Female, no.(\%) & $26(89)$ & $25(83)$ \\
AMA-positive, no.(\%) & $29(100)$ & $0(0)$ \\
UDCA treatment, no.(\%) & $24(83)$ & $0(0)$ \\
UDCA response*, no.(\%) & $12(41)$ & $0(0)$ \\
Cirrhosis, no.(\%) & $11(38)$ & $0(0)$ \\
\hline
\end{tabular}

the sole treatment at the time of enrolment. The participants were outpatients and inpatients from the Gastroenterology Department of Peking University Third Hospital from 2015 to 2016. All participants provided written informed consent. For each patient, an EDTA venous blood sample was collected and used for isolating plasma.

\section{RNA extraction}

For each sample, the total RNA from $2 \mathrm{~mL}$ of plasma containing EDTA anticoagulant was extracted by using TRIZOL reagent (Invitrogen, NY, USA) in accordance with the manufacturer's protocol; the total RNA was then quantified using a NanoDrop ND-1000 spectrophotometer (Thermo Fisher Scientific, Waltham, USA). The RNA integrity of each sample was assessed by denaturing agarose gel electrophoresis.

\section{CircRNA microarray labelling and hybridization}

RNA from the plasma of six PBC patients and six control subjects was extracted for microarray analysis; we pooled three samples together for the microarray assay to minimize patient heterogeneity. Total RNA from each sample was quantified using a NanoDrop ND-1000. Sample preparation and microarray hybridization were performed based on the standard protocol from Arraystar (Arraystar, Rockville, USA). Briefly, total RNA was digested with RNase R (Epicentre, Madison, USA) to remove linear RNAs and enrich circRNAs. Then, the enriched circRNAs were amplified and transcribed into fluorescent cRNA using a random priming method and an Arraystar Super RNA Labeling Kit (Arraystar, Rockville, USA). The labelled cRNAs were purified by using an RNeasy Mini Kit (Qiagen, Hilden, Germany). The concentration and specific activity of the labelled cRNAs (pmol Cy3/ $\mu \mathrm{g}$ cRNA) were measured by a NanoDrop ND-1000. A total of $1 \mu \mathrm{g}$ of each labelled cRNA was fragmented by adding $5 \mu \mathrm{l}$ of $10 \times$ blocking agent and $1 \mu \mathrm{l}$ of $25 \times$ fragmentation buffer; then, the mixture was heated at $60^{\circ} \mathrm{C}$ for $30 \mathrm{~min}$; finally $25 \mu \mathrm{l}$ of $2 \times$ hybridization buffer was added to dilute the labelled cRNA. A total of $50 \mu \mathrm{l}$ of hybridization solution was dispensed into the gasket slide and added to the Arraystar Human CircRNA Array V2.0 (8x15K) (Arraystar, Rockville, USA). The slides were incubated for 17 hours at $65^{\circ} \mathrm{C}$ in an Agilent Hybridization Oven. The hybridized arrays were washed, fixed and scanned using the Agilent Scanner G2505C.

\section{CircRNA microarray data analysis}

Scanned images were imported into Agilent Feature Extraction software (version 11.0.1.1) for raw data extraction. Quantile normalization of the raw data and subsequent data processing were performed using the $\mathrm{R}$ software package ( $\mathrm{R}$ version 3.1.2). After quantile normalization of the raw data, low intensity filtering was performed, and circRNAs that had flags in "P" or "M" ("All Targets Value") for at least 3 out of 6 samples were retained for further analyses. CircRNAs that were differentially expressed between the two groups were conveniently estimated by fold-change filtering and Student's t-tests. CircRNAs with fold changes $\geq 1.5$ and p-values $\leq 0.05$ were selected as significantly differentially expressed.

\section{Real-time PCR Analysis}

cDNA samples were prepared from $3.0 \mu \mathrm{g}$ of total RNA by reverse transcription using a Maxima First Strand cDNA Synthesis Kit (Fermentas, Hanover, USA) according to the supplier's instructions. In total, 8 candidate circRNAs were analysed by SYBR green I dye-based detection (Roche, Mannheim, Germany) with specific primer sequences (Table 2). The PCR conditions for candidate circRNAs and reference genes were 5 


\section{Cellular Physiology Cell Physiol Biochem 2017;44:1271-1281 \begin{tabular}{ll|l} 
DOI: 10.1159/000485487 & $\begin{array}{l}\text { O 2017 The Author(s). Published by S. Karger AG, Basel } \\
\text { www.karger.com/cpb }\end{array}$ \\
and Biochemistry Published online: November 29, 201
\end{tabular}

Table 2. Specific circRNAs primers for quantitative PCR analysis. (PS, product size)

\begin{tabular}{|c|c|c|}
\hline Name & Sequence & PS(bp) \\
\hline \multirow{2}{*}{$\beta$-actin(H) } & F:5' GTGGCCGAGGACTTTGATTG3' & \multirow{2}{*}{73} \\
\hline & R:5' CCTGTAACAACGCATCTCATATT3' & \\
\hline \multirow{2}{*}{ hsa_circ_402458 } & F:5' TGTATCTCCAGGGCAGCTTACT3' & \multirow{2}{*}{138} \\
\hline & R:5' TTGGATGGTCAGAAGGGTTTG3' & \\
\hline \multirow{2}{*}{ hsa_circ_052372 } & F:5' TCAATGCCCACAAGGACCAC3' & \multirow{2}{*}{56} \\
\hline & R:5' ACAGTACGTTCACCATCCCGAG3' & \\
\hline \multirow{2}{*}{ hsa_circ_407176 } & F:5' AAGACACTCTGCTTCTGCTGT3' & \multirow{2}{*}{78} \\
\hline & R:5' ATTTCAGGTCCAGAACTGGCT3' & \\
\hline \multirow{2}{*}{ hsa_circ_087631 } & F:5' GGATCGGGTAAAGATACCAGTG3' & \multirow{2}{*}{155} \\
\hline & R:5' GGCTGTCATTCCATCCTTGC3' & \\
\hline \multirow{2}{*}{ hsa_circ_102485 } & F:5' TGTGTACGAGATTCCGGTTGAGT3' & \multirow{2}{*}{144} \\
\hline & R:5' CGAGGTGGAAGGATCATTTGAG3' & \\
\hline \multirow{2}{*}{ hsa_circ_082319 } & F:5' GGGAGAATGGTGGAACTGAAC3' & \multirow{2}{*}{176} \\
\hline & R:5' AGAACTTCTCATGGGCAGTACAC3' & \\
\hline \multirow{2}{*}{ hsa_circ_000361 } & F:5' TCCATCCCAGTCCAGTTCCTAC3' & \multirow{2}{*}{156} \\
\hline & R:5' CACAGATGACCCTTCTTCCTCC3' & \\
\hline \multirow{2}{*}{ hsa_circ_406329 } & F:5' TTTGAGTTTTTAAAAGCCACCG3' & \multirow[b]{2}{*}{46} \\
\hline & R:5' GTTCTAAACAGTTATGGCTGGATG3' & \\
\hline
\end{tabular}

min at $95^{\circ} \mathrm{C}$ followed by 40 cycles of denaturation for $30 \mathrm{~s}$ at $95^{\circ} \mathrm{C}$, annealing for $30 \mathrm{~s}$ at $60^{\circ} \mathrm{C}$, and elongation for $30 \mathrm{~s}$ at $72^{\circ} \mathrm{C}$. qRT-PCR was performed on a LightCycler $480 \mathrm{II}$ real-time PCR system (Roche, Mannheim, Germany). The relative expression levels of circRNAs were determined by the $\Delta \mathrm{Ct}$ method and normalized to those of the housekeeping gene $\beta$-actin.

\section{Statistical analysis}

The results are reported as the mean \pm SEM. All experiments were performed and analysed in triplicate. The results from two groups were evaluated using unpaired t-tests. The clinical diagnostic value of a given circRNA was verified by ROC curve analysis, and when the AUC was equal to 0.5, the circRNA was defined as having no diagnostic value. $P<0.05$ was considered statistically significant. All statistical analyses were conducted using GraphPad Prism V5.0 software (GraphPad Software, San Diego, USA).

\section{Results}

CircRNA expression profiles in peripheral blood from PBC patients

Hierarchical clustering was used to determine circRNA expression in PBC patients and healthy individuals (Fig. 1A). Scatter and volcano plots show the variations in circRNA expression between the PBC patients and healthy individuals (Fig. 1B, C). A total of 7344 circRNAs were detected by the Arraystar Human CircRNA Microarray. We identified 22 circRNAs that were differentially expressed in the PBC patients versus the healthy controls. Eighteen of these circRNAs were up-regulated (Table 3), and 4 were down-regulated in the PBC patient plasma samples (Table 3). Among the up-regulated circRNAs, 13 were exonic, 3 were intronic, and 1 was antisense. All the down-regulated circRNAs were exonic. Screening criteria were utilized to obtain candidate biomarkers that would be most applicable in 


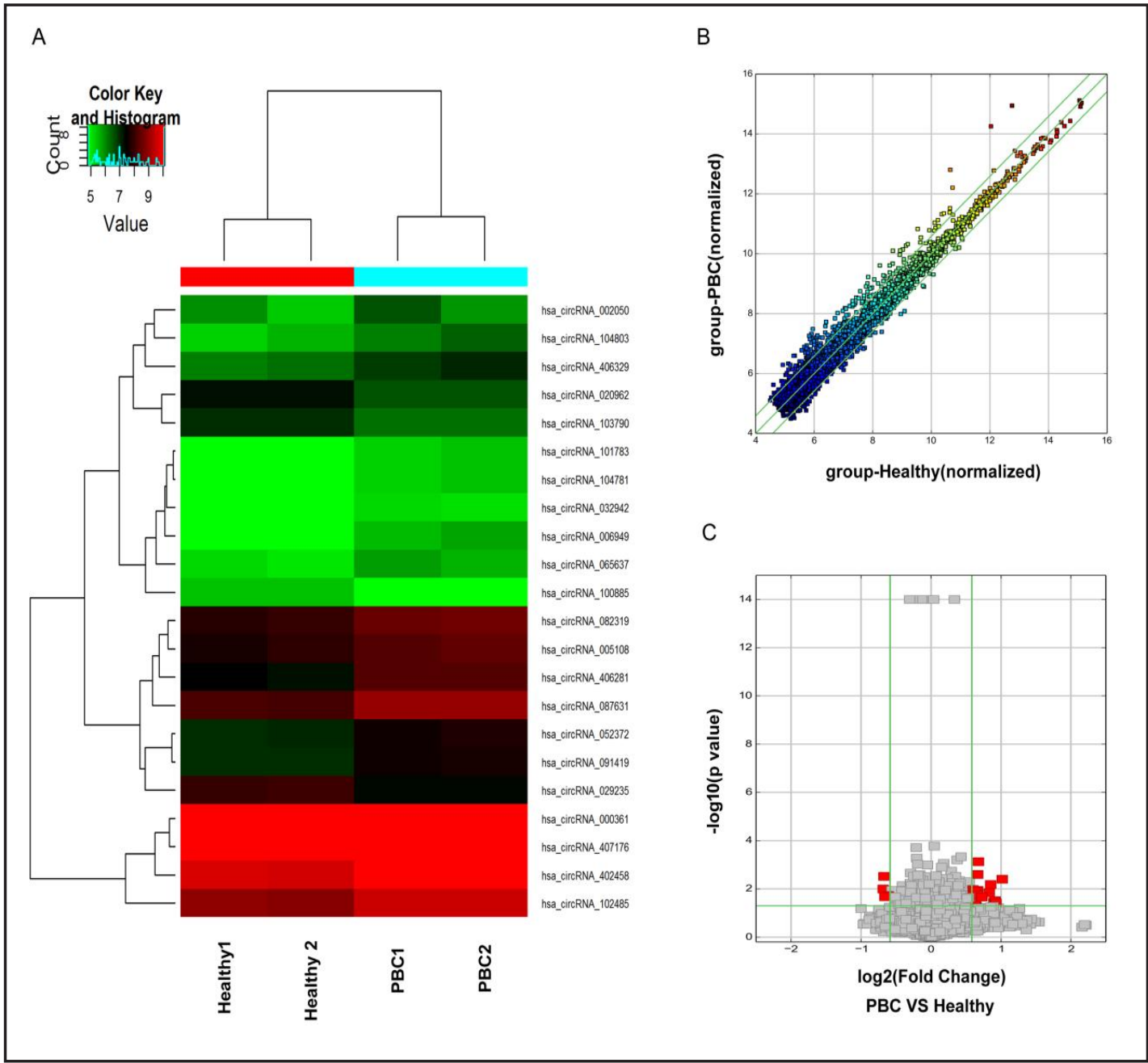

Fig. 1. Differential expression levels of circRNAs in plasma from PBC patients and healthy individuals. (A) Hierarchical clustering shows distinguishable circRNA expression profiling between PBC patients and healthy individuals. The dendrogram shows the relationships among the expression levels of the samples. Expression values are represented in different colours, indicating expression levels above and below the median expression level across all samples. (B) A scatter-plot was used to assess variations in circRNA expression levels between the PBC and healthy groups. The X and Y axes values in the scatter-plot are the normalized signal values of the samples ( $\log 2$ scaled). The circRNAs above the top green line and below the bottom green line were changed by more than 1.5-fold between the two compared groups. (C) Volcano plots were constructed using the fold change and $\mathrm{P}$ values. The horizontal line corresponds to a 1.5-fold upregulation and a 1.5-fold down-regulation, and the vertical line represents a P-value of 0.05 . The red point on the plot represents the differentially expressed circRNAs with statistical significance.

clinical practice: the fold changes were $\geq 1.5$, the $P$-values were $\leq 0.05$, and the raw intensity values were $\geq 200$ for each sample. 8 circRNAs (hsa_circ_402458, hsa_circ_052372, hsa circ_407176, hsa_circ_087631, hsa_circ_102485, hsa_circ_082319, hsa_circ_000361 and hsa_circ_406329) met these standards and were selected for subsequent verification by qRTPCR.

Expression profiles of circRNAs verified by $q R T-P C R$

To validate the circRNA microarray results, qRT-PCR was performed to analyse selected circRNAs. Although hsa_circ_102845 was not successfully amplified, the other 7 circRNAs showed consistent results between the qPCR and microarray analyses (Table 4, Table 3). All 


\section{Cellular Physiology Cell Physiol Biochem 2017;44:1271-1281

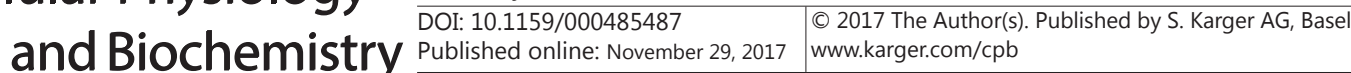

Table 3. Differentially expressed circRNAs between PBC and Healthy plasma.( FC, fold change)

\begin{tabular}{|c|c|c|c|c|c|c|c|c|}
\hline circRNA & P-value & FC (abs) & Regulation & $\begin{array}{l}\text { circRNA } \\
\text { type }\end{array}$ & chrom & strand & best_transcript & GeneSymbol \\
\hline hsa_circ_104803 & 0.00654706 & 1.8044287 & up & exonic & $\operatorname{chr} 9$ & - & NM_013438 & UBQLN1 \\
\hline hsa_circ_402458 & 0.0349801 & 1.8630203 & up & exonic & $\operatorname{chr} 2$ & + & NM_152523 & CCNYL1 \\
\hline hsa_circ_065637 & 0.04833133 & 1.5052161 & up & exonic & chr3 & - & NM_000581 & GPX1 \\
\hline hsa_circ_052372 & 0.00073809 & 1.5998668 & up & exonic & chr19 & + & NM_005762 & TRIM28 \\
\hline hsa_circ_006949 & 0.00395482 & 2.0224805 & up & intronic & chr1 & - & ENST00000311223 & GLUL \\
\hline hsa_circ_407176 & 0.03059671 & 1.8856876 & up & intronic & $\operatorname{chr} 9$ & + & ENST00000534928 & ZCCHC7 \\
\hline hsa_circ_032942 & 0.01151127 & 1.5861962 & up & exonic & chr14 & - & NM_004755 & RPS6KA5 \\
\hline hsa_circ_087631 & 0.0142703 & 1.7767426 & up & exonic & chr9 & + & NM_014282 & НАBP4 \\
\hline hsa_circ_104781 & 0.03300364 & 1.5508983 & up & exonic & chr9 & + & NM_012203 & GRHPR \\
\hline hsa_circ_002050 & 0.01045804 & 1.5157215 & up & exonic & chr17 & + & NR_034082 & LOC100130950 \\
\hline hsa_circ_102485 & 0.03233656 & 1.554524 & up & exonic & chr19 & + & uc002nir.1 & PGPEP1 \\
\hline hsa_circ_406281 & 0.03776612 & 1.8996156 & up & intronic & $\operatorname{chr} 3$ & + & ENST00000394799 & GNL3 \\
\hline hsa_circ_091419 & 0.01224217 & 1.5481588 & up & exonic & $\operatorname{chrX}$ & - & ENST00000361575 & RPL39 \\
\hline hsa_circ_005108 & 0.0462282 & 1.5137836 & up & exonic & chr14 & - & NM_203301 & FBX033 \\
\hline hsa_circ_101783 & 0.04542109 & 1.5436236 & up & exonic & chr16 & - & NM_015171 & XP06 \\
\hline hsa_circ_082319 & 0.00251391 & 1.5885285 & up & exonic & chr7 & - & NM_016478 & ZC3HC1 \\
\hline hsa_circ_000361 & 0.01752141 & 1.5825964 & up & antisense & $\operatorname{chr} 3$ & - & NM_015184 & PLCL2 \\
\hline hsa_circ_406329 & 0.02156069 & 1.6561258 & up & intronic & $\operatorname{chr} 3$ & - & ENST00000295633 & FSTL1 \\
\hline hsa_circ_100885 & 0.00994368 & 1.6076367 & down & exonic & $\operatorname{chr} 11$ & - & NM_002869 & RAB6A \\
\hline hsa_circ_029235 & 0.01860616 & 1.5304197 & down & exonic & $\operatorname{chr} 12$ & + & NM_020936 & DDX55 \\
\hline hsa_circ_103790 & 0.02092988 & 1.5797505 & down & exonic & $\operatorname{chr} 5$ & + & NM_005885 & MARCH6 \\
\hline hsa_circ_020962 & 0.00300402 & 1.5969763 & down & exonic & chr11 & - & uc001mai.1 & HBG2 \\
\hline
\end{tabular}

Table 4. Validation of some differentially expressed circRNAs using microarray samples by qRT-PCR( FC, fold change). $\mathrm{NA}=$ Not amplified

\begin{tabular}{|c|c|c|c|}
\hline circRNA & $\mathrm{PBC}(\mathrm{FC}, \mathrm{n}=2)$ & Healthy(FC,n=2) & PBC/Healthy \\
\hline hsa_circ_402458/ $\beta$-actin & 0.022 & 0.012 & 1.887 \\
\hline hsa_circ_052372/ $\beta$-actin & 0.028 & 0.025 & 1.120 \\
\hline hsa_circ_407176/ $\beta$-actin & 0.039 & 0.018 & 2.148 \\
\hline hsa_circ_087631/ $\beta$-actin & 0.030 & 0.016 & 1.848 \\
\hline hsa_circ_102845/ $\beta$-actin & NA & NA & NA \\
\hline hsa_circ_082319/ $\beta$-actin & 0.027 & 0.018 & 1.529 \\
\hline hsa_circ_000361/ $\beta$-actin & 0.027 & 0.024 & 1.141 \\
\hline hsa_circ_406329/ $\beta$-actin & 0.033 & 0.028 & 1.154 \\
\hline
\end{tabular}

the selected circRNAs exhibited significantly higher expression levels in the PBC patients than in the healthy individuals (Table 4). Five circRNAs, including hsa_circ_402458, hsa circ_407176, hsa_circ_087631, hsa_circ_082319 and hsa_circ_406329, were selected as candidate biomarkers for further validation in a large cohort. The expression levels of hsa circ_402458 $(P=0.0033)$ and hsa_circ_406329 $(P=0.0185)$ were significantly higher in the $\mathrm{PBC}$ group than in the healthy group; these results were consistent with the microarray data (Fig. 2). Hsa_circ_407176 $(P=0.0066)$ and hsa_circ_082319 had lower expression levels in the $\mathrm{PBC}$ group than in the healthy group; these expression patterns were opposite of the microarray data in the large validation cohort (Fig. 2). There were no significant differences in the expression levels of has_circ_087631 between the PBC patients and healthy controls (Fig. 2).

Moreover, we analysed the circRNA expression profiles in different groups according to different clinical characteristics (Fig. 3). We determined that hsa_circ_402458 expression 


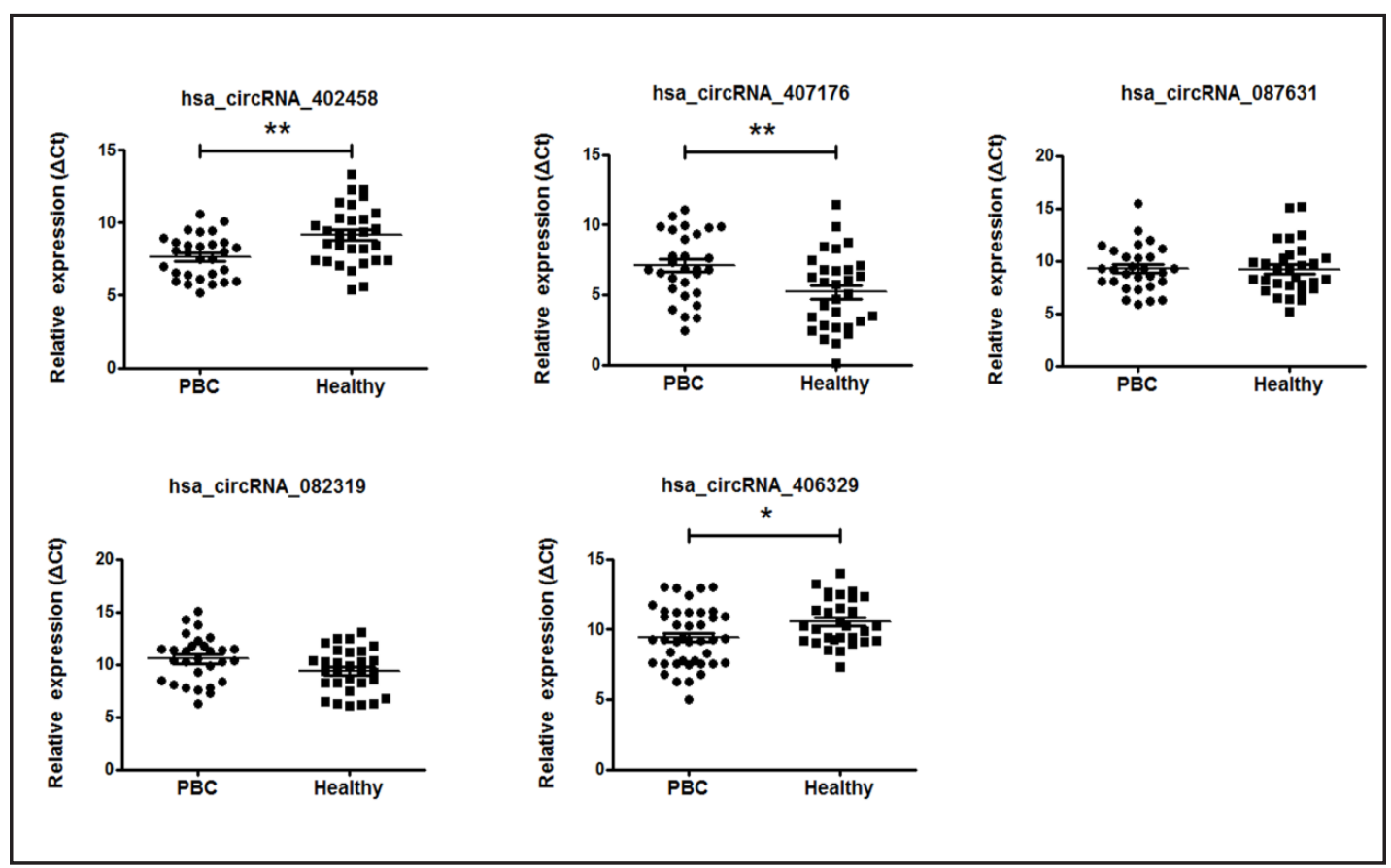

Fig. 2. Validation of the differential expression of candidate circRNAs. The expression levels of the following circRNAs were analysed via qRT-PCR (PBC: $n=29$, healthy: $n=30$ ): hsa_circ_402458, hsa_circ_407176, hsa_ circ_087631, hsa_circ_082319 and hsa_circ_406329. $\Delta$ Ct values were used to measure gene expression, which was normalized to the $\beta$-actin expression levels. The values presented are the means \pm SEM. ${ }^{*} \mathrm{P}<0.05$, $* * \mathrm{P}<0.01$.

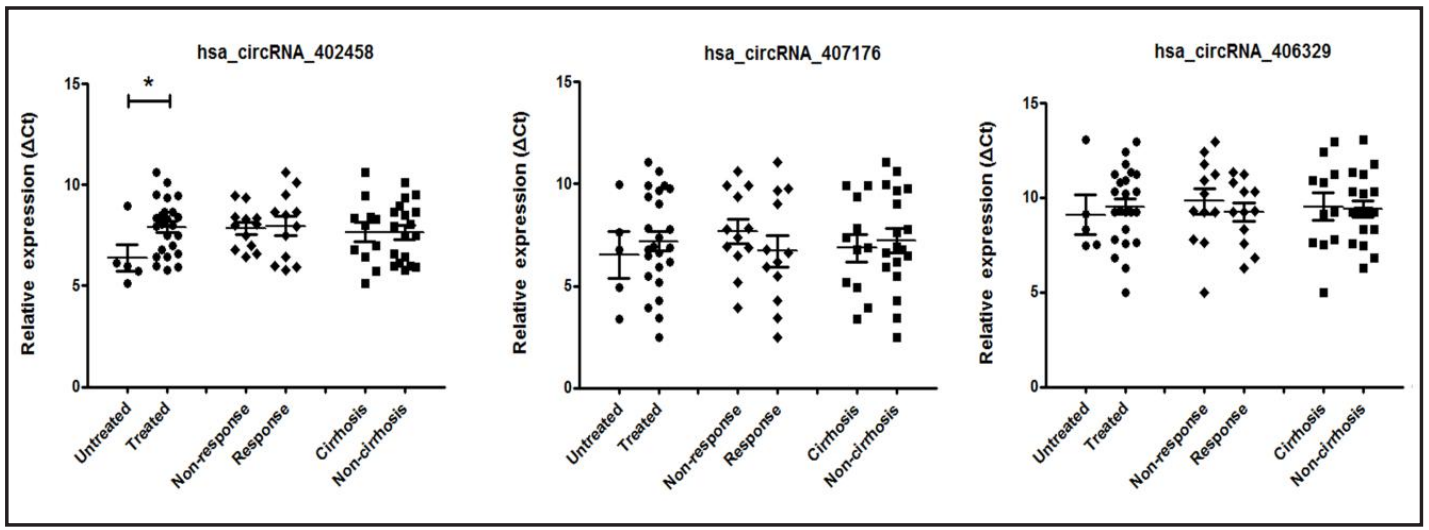

Fig. 3. Differential expression of circRNAs in different PBC subgroups. Expression levels of hsa_circ_402458, hsa_circ_407176, and hsa_circ_406329 in the three groups of PBC patients: patients not receiving UDCA therapy $(n=5)$ and patients receiving UDCA therapy $(n=24)$; UDCA non-responders $(n=12)$ and UDCA responders $(n=12)$; and patients with cirrhotic $P B C(n=11)$ and patients with asymptomatic $P B C(n=18)$. $\Delta \mathrm{Ct}$ values were used to measure gene expression, which was normalized to $\beta$-actin expression levels. The values presented are the means \pm SEM. $* * P<0.01$.

levels were significantly higher in $\mathrm{PBC}$ patients without UDCA treatment than in PBC patients receiving UDCA treatment $(P=0.0338)$. There were no obvious differences in expression levels in PBC patients with cirrhosis vs. asymptomatic or UDCA responders vs. non-responders. There were no significant differences in has_circ_407176 and hsa_circ_406329 expression levels among the three subgroups. Taken together, hsa_circ_402458 expression levels were significantly higher in PBC patients not receiving UDCA therapy than in patients receiving UDCA. 

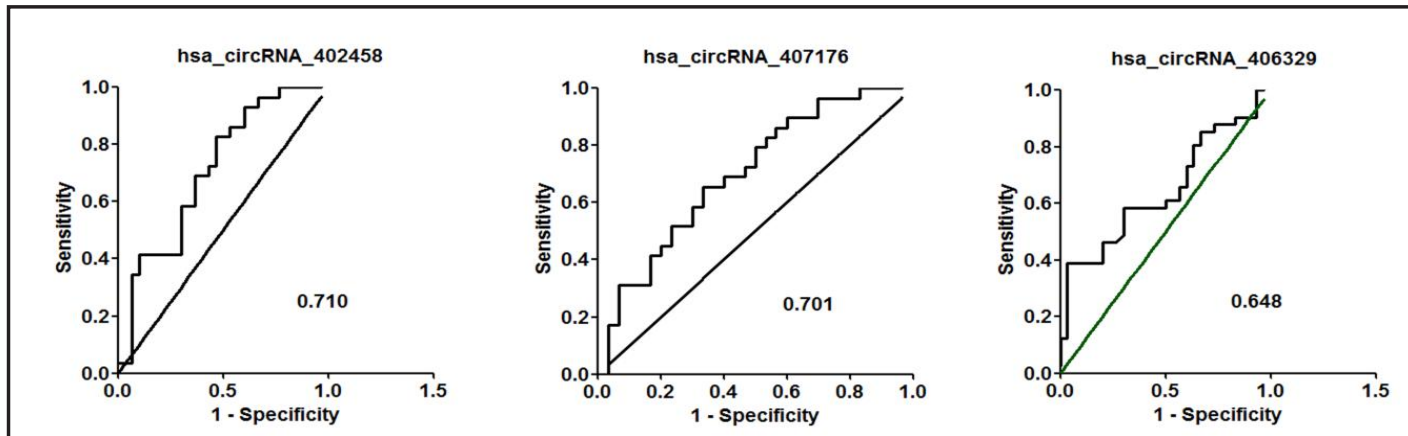

Fig. 4. ROC curve analyses of hsa_circ_402458, hsa_circ_407176, and hsa_circ_406329 for diagnosing PBC. The AUC values are given on the graphs.

Table 5.Validation of the selected circRNAs in a large cohort by qRT-PCR(PBC=29,Healthy=30)

\begin{tabular}{cccccc}
\hline circRNA & AUC & $95 \%$ CI & P value & Sensitivity & Specificity \\
\hline hsa_circ_402458 & 0.710 & $0.578-0.842$ & 0.005 & 0.690 & 0.633 \\
hsa_circ_407176 & 0.701 & $0.568-0.840$ & 0.008 & 0.655 & 0.667 \\
hsa_circ_406329 & 0.648 & $0.502-0.786$ & 0.057 & 0.586 & 0.700 \\
\hline
\end{tabular}

ROC curve analysis of differentially expressed circRNAs

The most significant differences in expression levels between the $\mathrm{PBC}$ and healthy groups were for hsa_circ_402458 $(P=0.0033)$, hsa_circ_407176 $(P=0.0066)$, and hsa_circ_406329 $(P=0.0185)$. These 3 circRNAs were subjected to ROC curve analyses to determine their diagnostic values for PBC (Fig. 4, Table 5). The AUC of hsa_circ_402458 for diagnosing PBC was 0.710 ([0.578-0.842], $P=0.005)$. The AUC of hsa_circ_407176 for diagnosing PBC was 0.701 ([0.568-0.840], $P=0.008)$. The AUC of hsa_circ_406329 for diagnosing PBC was 0.648 ([0.502-0.786], $P=0.057)$. The sensitivity and specificity values for the 3 circRNAs are also shown in Table 5. From the 3 groups of data, hsa_circ_402458 was chosen as the best performing $\mathrm{PBC}$ biomarker due to its higher AUC, lower $P$ value and higher expression levels.

\section{Discussion}

Primary biliary cholangitis (PBC) is a chronic inflammatory autoimmune disease that develops based on interactions between genetic and environmental factors. The first FDAapproved treatment for PBC was UDCA. Approximatively $30-40 \%$ of patients fail to respond to UDCA [22]. PBC is usually hard to detect in asymptomatic patients; they rarely visit hospitals to seek diagnosis and therapy until the disease has progressed to a more severe stage. What is worse, most asymptomatic patients become symptomatic and die or require a liver transplant within 5 years of not receiving the standard treatment $[6,7]$.

Current diagnosis methods for PBC are mainly based on the clinical manifestations, serum biochemical features, and histopathological characteristics of patients. Liver histology characterized by non-suppurative cholangitis and the granulomatous destruction of interlobular bile ducts is one of the objective criteria for confirming PBC diagnosis and stratification [23]. In addition, serum antibodies specific for PBC are the other pivotal biomarkers for making a PBC diagnosis. It is well known that AMAs, especially AMA-M2, are excellent markers with high specificity and sensitivity for the routine diagnosis of PBC [24]. AMAs are present in 95\% of PBC patients; however, 5\% of patients with PBC are AMAnegative [25]. Notably, AMA-negative PBC patients had an observably worse prognosis than AMA-positive PBC patients [25]. AMA-negative PBC patients had observably lower free survival rates for liver-associated complications, including liver transplant and death, than AMA-positive PBC patients [25]. However, AMAs are not ideal haematological molecular 


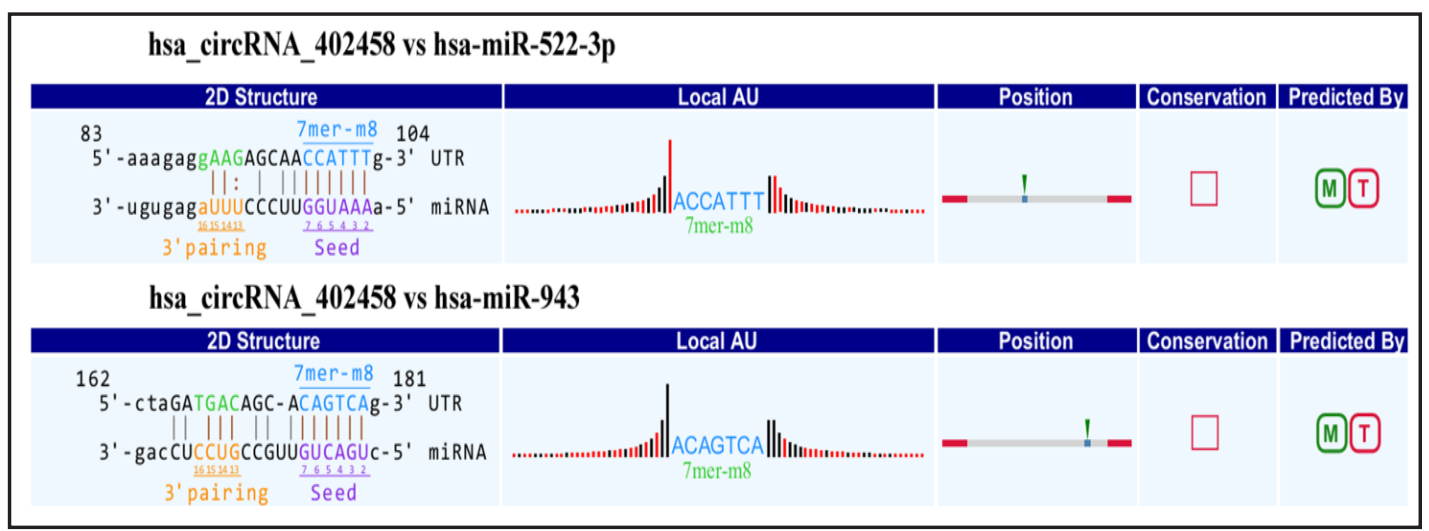

Fig. 5. Detailed annotation for hsa_circ_402458 and its potential miRNA target interactions. CircRNA/ microRNA interactions were predicted with miRNA target prediction software based on TargetScan [37] and miRanda [38].

markers for diagnosing PBC early, assessing its disease progression and determining treatment efficacy, especially for those AMA-negative PBC patients.

As a new class of non-coding RNAs, circular RNAs have attracted increasing attention from researchers. They may play an important role in gene expression and signalling pathways and participate in the development of human diseases [9, 26-28]. So far, there are few studies about the roles of circRNA in the pathogenesis of autoimmune diseases. Given the roles of miRNAs in the initiation and progression of autoimmune diseases and the roles of circRNAs in sponging miRNA and gene regulation, circRNAs may be associated with autoimmune disease initiation and progression and may be potential therapeutic targets for treatment. Only a few reports have reported dysregulated circRNA profiles in systemic lupus erythaematosus (SLE), multiple sclerosis (MS) and rheumatoid arthritis (RA), and certain circRNAs may serve as potential biomarkers for making a disease diagnosis [29-32]. For PBC, some long noncoding RNAs (lncRNAs) and miRNAs have been demonstrated to be involved in the occurrence and development of PBC and can be used as biomarkers for identifying PBC patients [33]. However, circRNAs have two significant properties: they are highly conserved and remarkably stable with a half-life $>48 \mathrm{~h}$. In addition to these properties, circRNAs can exist in exosomes and blood plasma because of their stability $[9,28]$. Consequently, circRNAs may be superior to miRNAs and IncRNAs regarding the diagnosis of human disease and could be applied to PBC.

To the best of our knowledge, this study is the first to investigate the expression profiles of circRNAs in peripheral plasma from PBC patients and to improve the understanding of the role of circRNAs in the pathogenesis of PBC. From the microarray analysis, we identified 22 significantly differentially expressed circRNAs-18 up-regulated and 4 down-regulated circRNAs in PBC patients versus healthy controls. We then choose 8 circRNAs according to the established criteria and validated them by qRT-PCR in a larger cohort. Hsa_circ_402458 showed the greatest significant difference in expression and the highest diagnostic value for PBC among the candidate biomarkers, suggesting that hsa_circ_402458 can be considered a promising diagnostic biomarker for $\mathrm{PBC}$ in clinical practice and that it may be comparable with traditional serum biomarkers. What is more, hsa_circ_402458 is expressed at significantly higher levels in PBC patients not receiving any drug therapy than in PBC patients receiving UDCA therapy, suggesting that hsa_circ_402458 may participate in the pathogenesis of PBC.

The field of circRNAs is quite new, and to the best of our knowledge, no studies have definitively demonstrated the bio-function of hsa_circ_402458. Recently, it was reported that circRNAs function as miRNA 'sponges' that naturally sequester and competitively suppress miRNA activity. Their interaction with disease-associated miRNAs indicates that circular RNAs are important for regulating diseases [13]. To determine the function of candidate biomarker circRNAs, we searched for potential miRNA targets of circRNA utilizing 


\section{Cellular Physiology Cell Physiol Biochem 2017;44:1271-1281 and Biochemistry Published \begin{tabular}{l|l} 
DOI: 10.1159/000485487 & (c) 2017 The Author(s). Published by S. Karger AG, Basel \\
www.karger.com/cpb
\end{tabular} \\ Zheng et al.: Hsa_Circ_402458 is Upregulated in Primary Biliary Cholangitis}

Arraystar's miRNA target prediction software. For hsa_circ_402458, two putative miRNAs targets, hsa-miR-522-3p and hsa-miR-943, were selected (Fig. 5). We speculated that hsa circ_402458 may target hsa-miR-522-3p and hsa-miR-943 and thus competitively suppress miRNA activity to participate in the pathogenesis of PBC. A previous study has demonstrated that miR-522-3p may be involved in the abnormal resolution of inflammation and may be a target for modulating chronic inflammatory disorders. Importantly, inflammation that is not resolved is a major driver of autoimmune diseases [34]. In addition, miR-943 was reported to be involved in the repair of DNA double-strand breaks through the TGF-beta signalling pathway. However, aberrant TGF- $\beta 1$ signalling contributed to the development of PBC in a murine model $[35,36]$. Therefore, we hypothesize that hsa_circ_402458 may function as a miRNA sponge and participate in the pathogenesis of PBC by influencing inflammationrelated signalling pathways. However, the potential function of these miRNA-circRNA axes in the pathogenesis of $\mathrm{PBC}$ requires further study.

Taken together, our study compared the expression profiles of circRNAs in plasma from PBC patients and matched control subjects by microarray analysis; then, we confirmed our findings in another larger case-control cohort. The results demonstrated that hsa circ_402458 is a sensitive and specific biomarker for diagnosing PBC and may be involved in the pathogenesis of PBC via interacting with target miRNAs. The precise molecular mechanisms underlying circRNA functions in PBC still require further investigation.

\section{Acknowledgements}

This work was supported by the National Natural Science Foundation of China (Grant No. 81400607) and the Natural Science Foundation of Beijing Municipality (Grant No. 7173274).

\section{Disclosure Statement}

The authors declare no conflicts of interest.

\section{References}

1 Selmi C, Bowlus CL, Gershwin ME, Coppel RL: Primary biliary cirrhosis. Lancet 2011;377:1600-1609.

$\checkmark 2$ Carey EJ, Ali AH, Lindor KD: Primary biliary cirrhosis. Lancet 2015;386:1565-1575.

3 Corpechot C, Chazouilleres O, Poupon R: Early primary biliary cirrhosis: Biochemical response to treatment and prediction of long-term outcome. J Hepatol 2011;55:1361-1367.

-4 Carbone M, Mells GF, Pells G, Dawwas MF, Newton JL, Heneghan MA, Neuberger JM, Day DB, Ducker SJ, Sandford RN, Alexander GJ, Jones DE: Sex and age are determinants of the clinical phenotype of primary biliary cirrhosis and response to ursodeoxycholic acid. Gastroenterology 2013;144:560-569.

5 Hirschfield GM, Mason A, Luketic V, Lindor K, Gordon SC, Mayo M, Kowdley KV, Vincent C, Bodhenheimer HJ, Pares A, Trauner M, Marschall HU, Adorini L, Sciacca C, Beecher-Jones T, Castelloe E, Bohm O, Shapiro D: Efficacy of obeticholic acid in patients with primary biliary cirrhosis and inadequate response to ursodeoxycholic acid. Gastroenterology 2015;148:751-761.

6 Mahl TC, Shockcor W, Boyer JL: Primary biliary cirrhosis: Survival of a large cohort of symptomatic and asymptomatic patients followed for 24 years. J Hepatol 1994;20:707-713.

7 Springer J, Cauch-Dudek K, O’Rourke K, Wanless IR, Heathcote EJ: Asymptomatic primary biliary cirrhosis: A study of its natural history and prognosis. Am J Gastroenterol 1999;94:47-53.

-8 Locke GR, Therneau TM, Ludwig J, Dickson ER, Lindor KD: Time course of histological progression in primary biliary cirrhosis. Hepatology 1996;23:52-56.

-9 Jeck WR, Sorrentino JA, Wang K, Slevin MK, Burd CE, Liu J, Marzluff WF, Sharpless NE: Circular RNAs are abundant, conserved, and associated with ALU repeats. RNA 2013;19:141-157.

10 Lasda E, Parker R: Circular RNAs: Diversity of form and function. RNA 2014;20:1829-1842.

11 Li P, Chen S, Chen H, Mo X, Li T, Shao Y, Xiao B, Guo J: Using circular RNA as a novel type of biomarker in the screening of gastric cancer. Clin Chim Acta 2015;444:132-136.

12 Jeck WR, Sharpless NE: Detecting and characterizing circular RNAs. Nat Biotechnol 2014;32:453-461. 


\section{Cellular Physiology Cell Physiol Biochem 2017;44:1271-1281 \begin{tabular}{l|l|l}
\hline DOI: 10.1159/000485487 & (c) 2017 The Author(s). Published by S. Karger AG, Basel
\end{tabular}

13 Salzman J: Circular RNA expression: Its potential regulation and function. Trends Genet 2016;32:309-316.

14 Conn SJ, Pillman KA, Toubia J, Conn VM, Salmanidis M, Phillips CA, Roslan S, Schreiber AW, Gregory PA, Goodall GJ: The RNA binding protein quaking regulates formation of circRNAs. Cell 2015;160:1125-1134.

15 Li Y, Zheng Q, Bao C, Li S, Guo W, Zhao J, Chen D, Gu J, He X, Huang S: Circular RNA is enriched and stable in exosomes: A promising biomarker for cancer diagnosis. Cell Res 2015;25:981-984.

16 Guarnerio J, Bezzi M, Jeong JC, Paffenholz SV, Berry K, Naldini MM, Lo-Coco F, Tay Y, Beck AH, Pandolfi PP: Oncogenic Role of Fusion-circRNAs Derived from Cancer-Associated Chromosomal Translocations. Cell 2016;166:1055-1056.

17 Legnini I, Di Timoteo G, Rossi F, Morlando M, Briganti F, Sthandier O, Fatica A, Santini T, Andronache A, Wade M, Laneve P, Rajewsky N, Bozzoni I: Circ-ZNF609 is a circular RNA that can be translated and functions in myogenesis. Mol Cell 2017;66:22-37.

18 Han YN, Xia SQ, Zhang YY, Zheng JH, Li W: Circular RNAs: A novel type of biomarker and genetic tools in cancer. Oncotarget 2017;8:64551-64563.

19 Zhao Z, Li X, Gao C, Jian D, Hao P, Rao L, Li M: Peripheral blood circular RNA hsa_circ_0124644 can be used as a diagnostic biomarker of coronary artery disease. Sci Rep 2017;7:39918.

20 Xu H, Guo S, Li W, Yu P: The circular RNA Cdr1as, via miR-7 and its targets, regulates insulin transcription and secretion in islet cells. Sci Rep 2015;5:12453.

21 Lukiw WJ: Circular RNA (circRNA) in Alzheimer's disease (AD). Front Genet 2013;4:307.

-22 Floreani A, Mangini C: Primary biliary cholangitis: Old and novel therapy. Eur J Intern Med 2017.

23 EASL Clinical Practice Guidelines: The diagnosis and management of patients with primary biliary cholangitis. J Hepatol 2017;67:145-172.

24 Hu S, Zhao F, Wang Q, Chen WX: The accuracy of the anti-mitochondrial antibody and the M2 subtype test for diagnosis of primary biliary cirrhosis: A meta-analysis. Clin Chem Lab Med 2014;52:1533-1542.

-25 Juliusson G, Imam M, Bjornsson ES, Talwalkar JA, Lindor KD: Long-term outcomes in antimitochondrial antibody negative primary biliary cirrhosis. Scand J Gastroenterol 2016;51:745-752.

26 Qian Y, Lu Y, Rui C, Qian Y, Cai M, Jia R: Potential significance of circular RNA in human placental tissue for patients with preeclampsia. Cell Physiol Biochem 2016;39:1380-1390.

27 Li H, Hao X, Wang H, Liu Z, He Y, Pu M, Zhang H, Yu H, Duan J, Qu S: Circular RNA expression profile of pancreatic ductal adenocarcinoma revealed by microarray. Cell Physiol Biochem 2016;40:1334-1344.

28 Meng S, Zhou H, Feng Z, Xu Z, Tang Y, Li P, Wu M: CircRNA: Functions and properties of a novel potential biomarker for cancer. Mol Cancer 2017;16:94.

-29 Li LJ, Huang Q Pan HF, Ye DQ: Circular RNAs and systemic lupus erythematosus. Exp Cell Res 2016;346:248-254.

30 Cardamone G, Paraboschi EM, Rimoldi V, Duga S, Solda G, Asselta R: The characterization of GSDMB splicing and backsplicing profiles identifies novel isoforms and a circular RNA that are dysregulated in multiple sclerosis. Int J Mol Sci 2017;18.

31 Li LJ, Zhao W, Tao SS, Leng RX, Fan YG, Pan HF, Ye DQ: Competitive endogenous RNA network: Potential implication for systemic lupus erythematosus. Expert Opin Ther Targets 2017;21:639-648.

-32 Ouyang Q, Wu J, Jiang Z, Zhao J, Wang R, Lou A, Zhu D, Shi GP, Yang M: Microarray expression profile of circular RNAs in peripheral blood mononuclear cells from rheumatoid arthritis patients. Cell Physiol Biochem 2017;42:651-659.

-33 Xie YQ Ma HD, Lian ZX: Epigenetics and primary biliary cirrhosis: A comprehensive review and implications for autoimmunity. Clin Rev Allergy Immunol 2016;50:390-403.

-34 Kang GJ, Lee HJ, Byun HJ, Kim EJ, Kim HJ, Park MK, Lee CH: Novel involvement of miR-522-3p in highmobility group box 1-induced prostaglandin reductase 1 expression and reduction of phagocytosis. Biochim Biophys Acta 2017;1864:625-633.

35 Danza K, De Summa S, Pinto R, Pilato B, Palumbo O, Carella M, Popescu O, Digennaro M, Lacalamita R, Tommasi S: TGFbeta and miRNA regulation in familial and sporadic breast cancer. Oncotarget 2017.

-36 Liu B, Zhang X, Zhang FC, Zong JB, Zhang W, Zhao Y: Aberrant TGF-beta1 signaling contributes to the development of primary biliary cirrhosis in murine model. World J Gastroenterol 2013;19:5828-5836.

-37 Enright AJ, John B, Gaul U, Tuschl T, Sander C, Marks DS: MicroRNA targets in Drosophila. Genome Biol 2003;5:R1.

-38 Pasquinelli AE: MicroRNAs and their targets: Recognition, regulation and an emerging reciprocal relationship. Nat Rev Genet 2012;13:271-282. 